\title{
Civilisations
}

Revue internationale d'anthropologie et de sciences

humaines

44 | 1997

Les peuples des forêts tropicales

\section{Frontières inter-ethniques au Choco et espace national colombien}

L'enjeu du territoire

Anne-Marie Losonczy

\section{(2) OpenEdition}

Journals

Édition électronique

URL : http://journals.openedition.org/civilisations/1623

DOI : $10.4000 /$ civilisations. 1623

ISSN : 2032-0442

Éditeur

Institut de sociologie de l'Université Libre de Bruxelles

Édition imprimée

Date de publication : 1 janvier 1997

Pagination : 138-151

ISBN : 2-87263-122-4

ISSN : 0009-8140

Référence électronique

Anne-Marie Losonczy, « Frontières inter-ethniques au Choco et espace national colombien »,

Civilisations [En ligne], 44 | 1997, mis en ligne le 29 juin 2009, consulté le 20 avril 2019. URL : http://

journals.openedition.org/civilisations/1623; DOI : 10.4000/civilisations.1623

(c) Tous droits réservés 
-

FAONTIËERES NTEER-ETHNOUUES

AU CHOCO ET ESPPCE MATIONAL

COLOMBEEN :

L'enjeu du territoire

Anne-Marie LOSONCZY 
La région du Choco colombien occupe une place particulière dans l'ensemble géographique et culturel que la littérature nomme «les basses terres tropicales sud-américaines». Cette contrée est couverte d'une forêt tropicale humide très dense de type «forêt-galerie», d'une richesse végétale plus importante que celle de l'Amazonie; elle est entrecoupée d'un nombre élevé de rivières et d'affluents dont le fond sablonneux recèle de l'or et du platine. Flle possède un littoral de plusieurs centaines de kilomètres, aussi bien au nord du Canal de Panamà (littoral Atlantique) qu'au sud (littoral Pacifique, plus étendu). Son climat est l'un des plus pluvieux au monde; on y enregistre en effet des précipitations annuelles qui varient entre 10.000 et $12.000 \mathrm{~mm}$ avec une humidité atmosphérique de $92 \%$ en moyenne. Le caractère écologiquement marginal de ce territoire par rapport aux autres zones tropicales de la Colombie et dans l'ensemble géographique du continent sudaméricain l'a voué à devenir une «zone de refuge» lieu d'une singularité culturelle et historique [West, 1957, Whitten, 1974 et Losonczy, 1992].

\section{F FORET PEUPPLEE}

Cette configuration écologique complexe, faite d'une forêt particulièrement dense et tenace, de lagunes, dilîts et de marécages côtiers et de cours d'eau aux courants capricieux et aux crues dévastatrices a contribué à empêcher la colonisation complète du Choco. Elle a freiné et retardé une pénétration hispanique, attirée pourtant par sà richesse aurifere, en y donnant naissance à un modèle d'exploitation absentéiste et partiel. Ce dernier est à l'origine de la présence presque exclusive dans la région de Noirs et de Mulâtres, descendants d'esclaves africains orpailleurs, à côté des petits groupes locaux d'Indiens Emberà et Waunanà, en position de repli en amont des fleuves et des affluents.

Après la guerre d'Indépendance qui met fin cutant à la domination espagnole qu'à l'institution de l'esclavage, la liberté de mouvement des esclaves noirs, qui désormais s'appelleront euxmêmes Libres, se traduit par un processus migratoire intense et généralisé qui donne lieu à un important brassage des populations noires pendant quelques décennies. Le Choco, outre un déploiement de sa population esclave propre sur les cours d'eau secondaires et tertiaires, reçoit un apport migratoire provenant essentiellement des zones minières du littoral Pacifique sud. La phlupart des Noirs, concentrés auparavant aux emplacements des grandes mines et aux centres de ramassage coloniaux du métal précieux, abandonnent ces lieux pour se disperser en petits groupes vers la partie basse des affluents et rivières non colonisés. Parallèlement, ceux parmi les Indiens que les missionnaires réussirent à fixer dans des poblados coloniaux, fuient également ces derniers vers les amonts des cours d'eau. Ce sera ce processus d'éparpillement post-colonial des deux groupes qui créera les conditions d'une seconde rencontre inter-ethnique, après celle qu'entraîna le régime de travail du début de l'époque coloniale.

La dissolution de la Colonie et les migrations qui s'ensuivent accentuent chez les Emberà et les Waunanà du Choco un parti pris d'atomisation sociale et d' 'solement par petits groupes de parentèle indépendants qui les caractérise dès la 
Conquête et qui constitue ensuite un dispositif efficace de résistance au régime de travail et d'évangélisation coloniaux. Le cérémonialisme culturel d'hostilité et d'alliances ponctuelles et changeantes, fondement de leur système guerrier traditionnel par raids, fut démantelé par l'action et la législation coloniales; la méfiance mutuelle intergroupes, qui lui a survécu, n'alimente désormais qu'un système de sorcellerie intra-ethnique, moyennant l'agression chamanique mutuelle (II $^{\text {I }}$ qui continue jusqu'à aujourd'hui à entretenir l'atomisation sociale et dialectale, de même que le soupçon réciproque.

\section{«LBPAES» ET EMAEFÀ :}

\section{territoires virtuels et ëchange}

Lhistoire du peuplement bi-ethnique de la rivière Capà ${ }^{[2]}$ constitue un exemple assez typique des processus similaires sur les autres cours d'eau secondaires et tertiaires du Choco après l' aholition de l'esclavage, même si le peuplement de cette rivière du Haut Choco est plus ancien et plus continu que celui de la plupart d'entre eux. Avec l'arrivée sur cette rivière des premières familles noires, apparaît un modèle doccupation de l'espace qui, outre l'unité fleuve-sol, comprend également le sol sous-aquatique, puisque les nouveaux Libres reprennent la pratique de l'orpaillage fluvial. Àcette époque, les maisons des Noirs s'égrènent le long de la rivière dans sa partie basse, à une journée de navigation de l'amont, alors que les maisons (tambo) emberà isolées occupent déjà le haut de la Capà.

Les effectifs des premiers petits groupes Noirs grossissent rapidement avec les nouvelles arrivées et de nombreuses naissances. Ils entreprennent le défrichage et la mise en culture d'abattis, choisis distants les uns des autres et ayant chacun deux à trois fois la dimension de la partie semée, de façon à pouvoir alterner jachère et semailles et pourvoir aux besoins d'une nombreuse descendance.

La recherche par les Noirs de terrains suffisamment élevés et près des berges des cours d'eau $^{[3]}$, celle de gisements auriferes riches, ainsi que la pratique de la chasse et les fréquents déplacements interfluviaux des Indiens mettent rapidement en contact les deux groupes. Ces contacts donnent lieu à divers échanges dont les initiateurs semblent avoir été les Noirs ici, comme ailleurs au Choco. Ces échanges consistent d'abord dans le troc de pirogues et de paniers de fabrication emberà contre des haches, des machettes, du sel. Plus tard, la gamme des biens échangés s'élargit et diverses modalités d'achat en argent complèteront le troc.

D'après les informateurs âgés des deux groupes, il semble que la présence indienne en amont de cette rivière - comme sur de nombreux autres cours d'eau du Choco - ait été légèrement antérieure à celle des Noirs, résultat peut-être des fuites, puis de la décomposition du poblado colonial indigène de Lloro, relativement proche.

Cette antériorité d'occupation de la rivière s'inscrit chez les Indiens dans la conscience d'une préséance sur le territoire, conscience enracinée dans les mythes et demeurée intacte pendant la période coloniale. Elle est cependant tempérée par le sentiment diffus d'une défaite, et par la volonté accrue de sauvegarder le parti pris culturel d'atomisation. Aussi les Emberà de l'amont acceptentils que la moitié basse de la rivière leur échappe car elle leur semble être encore trop proche des 
fleuves Atrato et Andàgueda, hauts-lieux de l'exploitation minière coloniale. En revanche, ils considèrent sa moitié haute comme faisant partie de la communauté Emberà, celle des vivants et des morts et ce non seulement dans sa partie exploitée par ses membres, mais aussi dans celle laissée en friche. Cette dernière leur apparât autant comme un réservoir nécessaire de chasse, d'horticulture et de coupe de bois qu'une garantie de la distance d'autres unités résidentielles; celle de l'indépendance par la dispersion.

Paradoxalement, on peut supposer que l'isolement et la méfiance intra-ethnique qui caractérisent les relations des petits groupes locaux emberà dispersés agissent dès cette période, dans le sens de la consolidation du système d'échange inter-ethnique avec les Noirs, mêne si celuj-ci constitue un pont tendu sur des sentiments collectifs diffus d'étrangeté et de dédain réciproques entre les deux communautés ${ }^{[4]}$.

L'exploitation, très intermittente, des emplacements fluviaux dorpaillage dans le haut de la rivière et de ses affluents par les Noirs ne semble pas avoir suscité l'opposition des Emberà; ces emplacements ont toujours été assez distants de leurs habitations et abattis. Mais cette acceptation tacite s'explique davantage par leur abandon radical de l'orpaillage, dès le début de la colonisation; aussi le sol sous-aquatique semble-t-il être devenu une partie non marquée de leur territoire.

En revanche, il en allait et il en va autrement pour les abattis que les Noirs ont défrichés près de l'amont; même distants de leurs habitations et terrains de chasse et laissés en friche, pour les Indiens ces lieux font partie d'une façon inaliénable du territoire emberà. Par conséquent, leur occupation et exploitation par les Noirs est considérée comme un prêt, don conditionnel, qui devrait donc appeler un échange compensatoire permanent de la part de ses bénéficiaires. Ce ne fut et ce n'est toujours pas le cas, car, en revanche, selon la conception noire, c'est le travail (de défrichage, d'exploitation et d'amélioration) qui engendre seul des droits d'usufruit, de transmission familiale et même de vente pour les ahattis, même si cette dernière ne peut être qu' intra-communautaire.

Quelles sont les raisons pour lesquelles ces deux représentations divergentes du rapport à la terre ont pourtant pu s'emboîter sans violence pendant plus d'un siècle? La première semble être une convergence sous-jacente entre elles. En effet, la pensée emberà n'établit, pas plus que la pensée noire, un lien d'inhérence, d'appartenance entre une portion délimitée du territoire et un groupe local. Pour les Indiens, le rapport entre ce dernier et un lieu riverain n'est pas pensé en termes d'appartenance réciproque figée mais plutôt en termes de marquage; autant par les rituels chamaniques, préalables à tout établissement de maisons et d'abattis, que par la mort et l'enterrement - même anciens - d'Emberà et notamment d'un chamane. Indépendamment de toute occupation ou usage pragmatique, ce marquage symbolique intègre définitivement le lieu dans une topographie emberà, virtuelle mais toujours actualisable. Celte symbolique de la virtualité territoriale se retrouve dans leur représentation des esprits chamaniques qui apparaissent comme des entités mouvantes, ambiguës et en constante métanorphose, flottant entre les différents niveaux de la cosmologie emberà et les différents lieux du territoire, entités dont l'immobilisation par un cha- 
mane est toujours pensée comme momentanée [Losonczy, 1990].

Cette territorialité virtuelle est portée par une réalité sociologique : les extrêmes fluidité et mobilité des groupes locaux emberà entre différentes rivières qui interdisent, elles aussi, toute représentation d'attachement définitif à une portion locale du territoire. Cette mobilité et capacité de scission des groupes reposent sur deux dispositifs sociaux traditionnels. I) abord, l'orientation bilatérale et classificatoire du système de parenté emberà, assortie d'une stratégie matrimoniale d'exogamie riveraine, engendre pour chaque Ego des droits d'usufruit et d'établissement sur plusieurs rivières, droits virtuels que l'on peut soit actualiser périodiquement ou durablement, soit laisser en suspens. En second lieu, l'une des modalités culturelles traditionnelles de résolution de conflits, à l'intérieur d'un groupe local de parentèle, est l'abandon de la rivière par une maisonnée et son établissement, momentané ou définitif, en amont d'un autre cours d'eau, parfois lointain.

Ainsi, la territorialité emberà se lit comme un ensemble de marquages rituels et sociaux qui créent un espace virtuel mouvant, largement indépendant de ses occupations pragmatiques: aussi, l'acceptation du défrichage noir de certains abattis de l'amont est-elle compatible avec sa perception comme «emberà».

La pensée et la praxis des Noirs, elles, marquent par le travail de défrichage ou de construction un territoire qui, à défaut de ces traces visibles, leur apparaît comme une sorte de no man s land. Ce travail crée des droits d'usutruit et de transmission familiale. Mais l'orientation également bilatérale de leur système de parenté et l'exogamie riveraine engendrent également pour les Ego des droits d'exploitation sur d'autres rivières, qui sont souvent laissés, momentanément ou à plus long terme, en suspens, mais sont toujours réactualisables. Les communautés noires ont également inscrit leur existence dans des cycles de mobilité familiale saisonnière et de mobilité résidentielle des individus, dont le support est une représentation de supralocalité cbocoana en termes d'une parenté diffuse de tous les achocoanos" noirs. Ainsi, la territorialité des Négro-Colombiens comporte également une part de virtualité représentée et vécue qui refuse l'inhérence à une portion de territoire délimitée et clôturée.

Cette convergence partielle sous-jacente entre deux représentations de territorialité a maintenu jusquu'à présent les limites territoriales inter-ethniques au Choco, comme frontières mouvantes, ouvertes à la modification, autour de noyaux stables d'habitation et d'usage domestique de l'espace, appartenant exclusivement à chacun des groupes. Mais leur divergence a constitué la scène d'un premier «malentendu» dans l'échange interethnique : la non-reconnaissance d'un don conditionnel des Indiens de la part des "preneurs" Noirs, qui, eux, s'estiment premiers, c'est-à-dire occupants d'une terre vide. Jusqu'à maintenant, l'abondance relative des terres, ainsi que les autres échanges entre les deux groupes ont ajouté leurs effets à la convergence partielle des représentations territoriales pour contenir une solution violente de ce conflit latent d'interprétations et de praxis.

Cet arrière-fond de réciprocité escamotée modèle à son tour les autres échanges, qui tendent à le compenser. Il s'agit notamment, au-delà des biens et services, de la circulation constante de 
«biens symboliques» entre les deux groupes : celle de noms propres, moyennant le système de parrainage inter-ethnique, celle d' un parler inter-ethnique, compromis entre l'ethnolecte noir du Choco et la langue emberà, et celle de représentations, d'items rituels et de procédés thérapeutiques. Cet ensemble systématique d'échanges crée une sorte d'espace social d'emboîtement entre les deux groupes [Losonczy, 1992] qui manifeste et met en forme la participation limitée dans la culture et les valeurs de l'autre. Toutefois, l'antagonisme latent peut parfois, moyennant l'intervention intempestive de la logique extérieure de l'Église ou de l'État, amener le système d'échange vers un dangereux point de rupture devant lequel. néanmoins, Noirs et Indiens ont toujours reculé jusqu'à présent.

Ies échanges de nature économique, établis au fil du temps entre les deux groupes ethniques, font l'objet d'une codification assez rigide quant aux produits échangés et aux modalités de ces échanges. En effet, les Indiens vendent aux Noirs deux types de biens : d'abord, une partie du surplus horticole (maïs et bananes) et parfois des poulets et des œufs. Ces ventes se réalisent surtout au moment du retour des Noirs de leur grande migration annuelle vers les zones d'orpaillage lointaines, moment où ils se trouvent momentanément à court de ces aliments. En second lieu, les produits de l'artisanat emberà comme les pirogues, taillées par les hommes - d'une importance capitale pour les Noirs dont peu savent les fabriquer - et les paniers utilitaires, fabriqués par les Indiennes, sont vendus à l'occasion des descentes des Fimberà dans le village noir pendant les trois périodes de fêtes communautaires, la
Semaine Sainte, la Saint-Antoine et Noël. Ces descentes n'ont pas que la vente comme motivation, car elles associent également les Indiens à ces fêtes. Ieur accueil donne lieu à un comportement assez formalisé d'hospitalité qui contraste dans une grande mesure avec celui de la convivialité intra-ethnique qui caractérise chacun des deux groupes.

Parallèlement, les Indiens achètent au village noir tous les produits de fabrication extérieure qu'ils ont peu à peu incorporés à leur existence sociale : sel, allumettes, kérosène, haches, machettes, fusils de chasse, harpons, eau-de-vie de fabrication régionale. En ce sens, la communauté noire se place comme la rectistributrice privilégiée d'objets manufacturés vers la société emberà, ce qui évite dans une grande mesure aux Indiens le contact avec les commerçants blancs de Quibdo. Les Noirs s'interposent entre les Emberà et la logique marchande brutale de la société nationale; par ce contact médiatisé et atténué avec cette logique, les Indiens ont pu faire un apprentissage moins traumatisant et meurtrier de certaines règles de l'univers blanc. Le rôle «tampon» des Noirs et l'expérience ainsi obtenue ont certainement contribué à la survie physique et culturelle des Indiens depuis la fin de l'époque coloniale.

Les Emberà ne vendent pas aux Noirs tout leur surplus agricole; ils prévoient toujours une réserve de mais pour les cas d'inondation, de mauvaises récoltes ou pour la fabrication cérémonielle de la bière de maïs (chicha). L'utilisation d'outils métalliques ne semble pas avoir augmenté leur production des biens de subsistance; en revanche, elle leur a fait gagner un temps qu'ils consacrent à des activités non productices 
de biens telles que baignades, beuveries, palabres, voyages. Par ailleurs, les produits de la chasse et de la pêche ne sont jamais échangés dans le réseau inter-ethnique : pour les premiers, it s' agit d'une activité symboliquement et pragmatiquement complémentaire pour l'homme noir, alors que c'est par elle que l'homme emberà acquiert du prestige social et affirme sa virilité; son produit doit être intégralement distribué parmi les membres de l'unité résidentielle. Pour ce qui est de la pêche, aucun surplus ne peut en être constitué à cause de la difficulté de conservation; d'autre part, le caractère très facilement et continuellement accessible de ce produit lui fait perdre sa valeur sociale.

Cette modalité codifiée d'échange inter-ethnique porte sur des biens liés à la subsistance et se réalise en termes de vente-achat. Ses temps forts sont liés à ceux du cycle des fêtes collectives des Noirs; son lieu est l'espace communautaire de ces derniers. Ce système maintient les interactions de circulation de biens à un niveau constant, sans débordements. Il n'admet qu'une exception, mais elle est récurrente : l'échange en termes de don de cadeaux qui scelle le parrainage, compadrazgo, lien de consanguinité rituelle, établi, moyennant le baptême d'un enfant emberà, entre un couple de Noirs et les parents de l'enfant.

Pendant la période de semailles et de récolte de maïs, les Noirs - souvent en mal de maind'œuvre familiale, en raison des activités concomitantes d'orpaillage - ont tendance à faire appel aux Indiens pour qu'ils les aident dans les abattis près de l'amont de la rivière. Ils leur offrent soit un salaire journalier, soit une quantité déterminée des produits de l'abattis par jour de travail, soit encore leur propre force de travail pour leur prêter main-forte à leur tour dans les travaux horticoles. L'argent ne tente pas les Indiens qui, grâce aux sommes reçues pour leurs pirogues et paniers, en ont plus que suffisamment pour leurs achats. Les produits horticoles ne les intéressent pas davantage : leur effectif démographique étant beaucoup plus réduit, ils en produisent toujours assez; la proportion d'adultes étant chez eux plus importante, ils n'ont pas besoin de la force de travail des Noirs pour faire face à tous les travaux de subsistance. Du reste, accepter de travailler avec d'autres que des co-résidents se trouve loin de leur conception de l'activité productrice qui est postulée uniquement comme lien familial ou communication individuelle avec un espace. Dès lors, cette acceptation revêt à leurs yeux le caractère d'un service extraordinaire que le salaire, en argent ou en nature, ne compense pas. Par conséquent, pour équilibrer l'échange, ils seront amenés à demander à Jeur tour des biens ou des services extraordinaires en plus (médicaments, cigarettes, tissus, poste de radio à piles par exemple). Les Noirs ne comprendront ni ne satisferont ces demandes, ce qui dissuade, une fois de plus, les Indiens à échanger leur travail et les Noirs à le leur demander. L'échange de services se trouve donc, à ce niveau, remarquablement freiné.

En résumé, tout se passe comme si, au fil du temps, dans le déploiement des échanges de biens et de services liés à la subsistance, la pratique et les représentations de chaque groupe ethnique en cause s'étaient emboîtés peu à peu pour équilibrer deux forces contraires : celle qui pousse à l'échange et celle qui aussitôt en limite le champ 
et la portée. Cet équilibre dynamique garantit autant leur quant-ìt-soi qu'un certain degré de relations.

Ceci fut, nous semble-t-il, facilité par deux similitudes d'ordre culturel qui rapprochent ces groupes géographiquement contigus depuis longtemps. Il s'agit, d'une part, de la similitude entre les technologies productives et, d'autre part, de celle qui, dans chacune de ces sociétés, frappe l'accumulation individuelle et même familiale de biens par le signe de la négativité sociale, même si les représentations idéologiques respectives de celle-ci diffèrent sensiblement d'un groupe à l'autre ${ }^{[5]}$. Mais il existe aussi une différence qui trace les limites entre les deux ensembles ethniques : celle matérialisée par ce filon d'or, brillant dans le sable des berges du Choco, qui n'a pas fini de fasciner les Noirs dont le déracinement et l'esclavage lui durent pourtant beaucoup. Son exploitation en revanche fut radicalement abandonnée par les Indiens dont les ancêtres l'ont découvert les premiers.

\section{CONTHWUTÉ ET WHOATTION:}

\section{les frontières inter-ethniques}

I. visage actuel du Choco rural est donc celui de la coexistence de deux groupes ethniques d'un poids démographique inégal. Cette coexistence, basée sur une rigoureuse séparation spatiale et sexuelle, a cependant engendré peu à peu un système d'échanges inter-ethniques dense et cohérent, néanmoins non dépourvu de points de conflit. Ce système se déploie autant dans les activités liées à la subsistance que dans les représentations culturelles et le rituel. Cette relation interethnique, d'abord asymétrique et puissamment médiatisée par la classe dominante de la société coloniale, tend ensuite à une certaine symétrie; mais elle n'est jamais entièrement indépendante des déchirements de l'histoire nationale. Ces avattars historiques permettent cependant de saisir le cheminement par lequel pour ces deux groupes l'existence spatiale et socio-culturelle de l'autre en est venue à signifier sa propre périphérie et ses propres limites.

Létat actuel des deux sociétés et de leurs rapports est le résultat d'un équilibre dynamique entre continuité et rupture par rapport à la société esclavagiste coloniale qui les a réunis par la force au Choco. La reprise autonome par les Noirs de forpaillage fluvial avec les procédés technologiques pré-coloniaux ${ }^{[5]}$, le désintérêt persistant des Emberà, face à cette activité, la séparation spatiale et sexuelle des deux groupes, le sentiment diffus et réciproque de suspicion mutuelle et l' «invisibilité» persistante de la population noire dans la conscience nationale colombienne; voilà les lignes de force d'une continuité entre le passé colonial et le présent. Mais I'utilisation inter-ethnique stratégique de cet héritage comme pièce-maitresse d'un système de communication qui garantit les limites de chaque identité ethnique et canalise les conflits, signale les brèches d'une rupture et les contours d'une innovation dans cette continuité.

Les processus qui constituent une rupture sociale entre l'époque coloniale et le présent affectent les deux groupes. les migrations et le brassage de populations noires, en quête de nouveaux territoires à investir, ont donné naissance au Choco à un peuplement dispersé qui sous-tend l'existence sociale des nouvelles communautés négro-colombiennes. Chez les Emberà, des chan- 
gements probables dans le système de parenté et l'apparition récente d'une organisation politique régionale pour la défense de l'autonomie indienne, organisation dont l'existence est censée faire contre-poids à l'atomisation et à la méfiance intra-ethniques; voilà les principaux axes de discontinuité par rapport à la période coloniale.

\section{ETHNSATION ET HISTOACLIEE:}

\section{le défi de la modernité}

En effet, l'on peut considérer que l'événement le plus novateur de l'histoire indienne du Choco durant ces dernières décennies a été la création de I'OREWA (Organizacion Regional EmberàWaunanà del (boco) en 1982. Fondée par d'anciens éièves emberà des Internats Indigènes régis par des missionnaires et soutenue à ses débuts par l'aile progressiste de l'ordre clarétien du Choco, l'Organizacion a su gagner rapidement son indépendance et s'est constituée en un coriace interlocuteur des autorités gouvernementales pour la «défense de la terre, la culture traditionnelle et l'autonomie» indiennes : depuis quelques années, elle fait partie également de l'Organizacion Nacional Indigena de Colombia (ONIC).

Par un paradoxe incontournable et désormais classique, l'organisation ne peut lutter pour cet objectif dans l'espace politique national que par des moyens politiques et idéologiques issus de la modemité. Elle le fait sous la direction de jeunes leaders indiens dont la connaissance de la langue et de la culture d' origine est souvent alussi limitée que teintée d'ambivalence, en raison de leur longue éducation dans les internats missionnaires et du contact continu avec des "assesseurs» (conseillers) de l'organisation, universitaires issus de la gauche urbaine. LOREWA a élaboré un programme scolaire d' "eéducation bilingue et bi-culturelle» et un programme de "promotion et d'éducation sanitaire», moyennant l'alphabétisation et la formation de quelques jeunes Emberà comme éducateurs et promoteurs de santé de leur groupe résidentiel.

En vue d obtenir des droits collectifs territoriaux. sanitaires et éducatifs de la société nationale, cette organisation fut amenée - avec un succès mitigé et limité jusqu'à maintenant - à tenter de vaincre l'atomisation séculaire et la dispersion résidentielle extrême des groupes locaux emberà, de même que leur isolement volontaire et leur mobilité incessante, en les incitant à se regrouper en "communautés» de type villageois. Elle tente également d'introduire dans ces groupes politiquement acéphales une structure d'autorité hiérarchique empruntée à la tradition hispanique coloniale : le "cabildo", censé fonctionner ici comme son relais local.

Parallèlement, les documents écrits, produits par l'organisation et largement diffusés par son programme d'éducation, s'engagent dans la construction d'une «histoire emberà», fondée sur le postulat d'une unité culturelle, territoriale, ethnique et politique immémoriale, base de la résistance au colonisateur. Cette construction ignore autant les systèmes de représentation de soi des groupes emberà que leurs extrême mobilité et atomisation linguistique, territoriale et organisationnelle. atomisation traditionnellement fondée sur une structure d hostilité guerrière intra-tribale et inter-tribale, documentée dés le début de la Colonie (razzias et embuscades périodiques de petits 
groupes d'alliance instable entre eux). Cette stratégie guerrière dispersée fut à plusieurs reprises déployée contre le colonisateur, avant d'être relayée par celle de la fuite vers les amonts des cours d'eau et par un chamanisme d'agression entre groupes locaux [Isacsson, 1974; Losonczy, 1986].

Ainsi, la pratique et la pensée de l'OREWh face aux groupes emberà semblent promouvoir un processus d' «ethnisation", assortie de son "historisaltion" rétrospective. Cette dernière est censée créer à la fois sa base de légitimation interne et exteme. Entamé face au défi que la modernité et la société nationale posent à l'autonomie indienne, ce double processus semble puiser paradoxalement dans les objectifs et outils institutionnels et idéologiques des missionnaires et des autorités civiles de la Colonie, contre lesquels la résistance des Emberà fut efficace, précisément en raison de leur atomisation et de leur mobilité.

\section{TEPRITORIRLITÉS IDÉOLOGIOUUES ET TERBITOHBES VIATUELS}

Devant l'enjeu de la modernité et de l'espace politique national, les groupes noirs ruraux du Choco, eux, ont continué à penser et à vivre leur identité sans référent ethnique et sans lutter pour des droits collectifs, territoriaux ou autres en tant que "communauté". En revanche, l'État colombien, après un débat politique passionné, s'est donné récemment (1991) une nouvelle constitution qui tranche sur le centralisne unificateur traditionnel de l'époque républicaine, en définissant la Colombie comme "multiculturelle et pluri-ethnique» et en proposant un statut reconnu de «minorité ethnique» aux sociétés indiennes, sta- tut assorti de droits territoriaux et culturels collectifs [Gros, 1991].

Ce nouveau statut pousse ces demières à parachever le processus d' «ethnisation», moyennant la création et la fixation de limites territoriales immuables et de structures internes d'autorité qui sont contraires à la logique culturelle traditionnelle de nombre d'entre elles, mais qui constituent la seule garantie de leur inclusion dans l'espace social et politique de la nation.

Au Choco, ce processus est inégalement entamé parmi les quelque 180 groupes locaux emberà et waunanà recensés et les résistances semblent souvent considérables devant l'autorité hiérarchique interne nouvelle et les directives de regroupement et de fixation des habitats. Ces dernières tentent précisénent d'établir un lien d'inhérence entre un territoire aux limites figées et immuables et un groupe dont les limites sociales seraient également soustraites à la mobilité. La logique d'un tel lien s'oppose précisément au caractère virtuel de la territorialité et de l'organisation sociale et symbolique emberà.

Parallèlement, certains groupes d'intellectuels noirs ont engagé récemment une bataille légale, couronnée de succès, pour que les communautés noires du Choco soient également reconnues conmme minorité ethnique et puissent donc légalement défendre des droits territoriaux collectifs, face à ceux des Indiens. Cette victoire a suscité dans la plupart des groupes urbains et ruraux des réactions de rejet, teintées cependant d'un certain réalisme : l'auto-définition en termes de «minorité ethnique noire» leur semble dépourvue de sens («nous sommes chocoanos»), ou «raciste» («Les Indiens, on ne les appelle pas par 
leur couleur»), les droits communautaires et non familiaux sur la terre leur semblent également inconcevables et limitatifs, autant de la transmission familiale que de la mobilité régionale qui est un des piliers de leur "être chocoano". Toutefois, le nouveau statut juridique et territorial des Indiens engendre une conscience diffuse du caractère inéluctable d'un changement dans le régime de territorialité propre.

Ainsi se trouve engagé au Choco un processus largement exogène et paradoxal de durcissement des limites identitaires en termes ethniques, parallèle à une fixation des limites territoriales entre groupes, jusqu'à présent floues et mobiles. Il s'inscrit dans un contexte de démocratisation réelle de la société nationale, fondée sur la nouvelle constitution, qui garantit et élargit les droits de l'individu, tente de limiter la corruption et de moderniser l'appareil administratif et judiciaire de l'État. Ce processus de durcissement identitaire ethnique est concomitant à l'entrúe indiesne dans un régime d'historicité de type «moderne», moyennant une tentative «savante» de reconstruction de la tradition, articulée sur un nouvel outil intellectuel : l'écriture. Les prémisses du même processus s'observent déjà dans des productions écrites de certains mouvements politico-intellectuels noirs. Celles-ci tentent la reconstitution d'une identité culturelle "afro-colombienne" autour de deux grands items historiques : l'Afrique des origines et l'esclavage. Or, c'est précisément sur l'oubli et le non-dit de ces deux items que s'est constituée la mémoire collective populaire des Négro-Colombiens qui s'auto-dénomment Libres.

À n'en pas douter, c'est l'historicité de la nation, ainsi que son espace idéologique, politique et institutionnel qui constituent le lieu d'emprunt de ces processus. Cet ensemble d'outils symboliques ne s'est trouvé disponible pour les sociétés du Choco que par le rapprochement économique et politique récent de l'śtat et de la région. Ceci conduit à penser que le long désintérêt post-colonial de l'État colombien face à cette dernière a également concouru à consolider le système d'interface syncrétique entre Noirs et Emberà dont la logique de mobilité et d'emboîtement résiste encore victorieusement sur les rivières.

\section{L'NWDEN. FGUUAE DE FAOHTIËAE DE L'ESPACE NATIONAL}

Ce constat de désintérêt persistant de l'État invite à s'interroger sur les caractéristiques de la territorialité dans l'idéologie nationale colombienne traditionnelle. La lecture des manuels d'histoire et de quelques documents politiques majeurs des cent dernières années [Triana, 1987] permet de valider pour la Colombie un constat fait a partir de l'examen du patriotisme hongrois [Losonczy \& Zempléni, 1991], à savoir que le territoire d'appartenance patriotique ne coïncide pas nécessairement avec les frontières légales de l'État. L'idéologie patriotique colombienne - contrairement à celle des Hongrois - a postuléet vécu ce territoire comme étant plus réduit que celui de l'État. Ainsi en a-t-elle symboliquement amputé la région amazonienne, la côte Pacifique et une partie des basses terres de l'Orient - régions forestières et marécageuses, peuplées de groupes indiens et noirs. Si, en 1824, un décret de la jeune République, portant sur «l'évangélisation et la pacification des tribus indiennes » parle de "pays limitrophes» en se référant à l'Amazonie, à l'Oré- 
noque et à la presqu'île de la Guajira qui font partie du territoire de l'État colombien [cité par 'Triana, op. cit. ], le manque ultérieur presque complet dans ces régions d'institutions nationales telles que la police, la mairie, l'armée, y compris dans les zones frontalières, ainsi que leur statut d' «intendance" (qui s'oppose à celui de «département » pour l'intérieur du pays), marquent une remarquable continuité historique dans cette réduction symbolique et pragmatique du territoire patriotique. En revanche, le réservoir d'auto-représentation de l'identité nationale se trouvait jusqu'à aujourd'hui dans les régions centrales andines du pays : c'est leur histoire qui était tenue exclusivement comme étant celle de la Colombie, ce sont certains de leurs items culturels qui sont emblématisés comme symboles nationaux et c'est d'elles que sont originaires tous les politiciens de stature nationale de l'époque républicaine.

Or, ces régions furent avant la Conquête le centre de la civilisation Muisca, faisant partie de l'ensemble Chibcha. Pourtant, contrairement au Pérou et au Mexique, l' «Indien seigneurial" chibcha semble relégué dans une temporalité préhistorique et se trouve donc singulièrement absent des emblèmes et des symboles de la nation colombienne «historique» dont la légitimité est construite sur une identité criolla (créole) plus tardive. Cette identité se revendique comme «métisse " mais son axe culturel organisateur est constitué par l'héritage hispanique. De la lecture des documents politiques et des manuels d'histoire se dégage une image floue des Chibcha; image de «nobles perdants", rapidement subnergés par un processus triomphant de métissage avec l'Espagnol dans lequel leur héritage apparaît davantage de l'ordre du "sang" que de celui de la "culture ». Cette image de perdants culturels, vite assimilés, a empêché dans cette idéologie patriotique l'émergence de l'Indien seigneurial, autant comme figure maitresse d'un «indigénisme " andin que comme paradigme-pivol d'une identité nationale.

Ainsi, l'idéologie nationale traditionnelle en Colombie a érigé l'Indien en une figure de frontière. Si l'Indien des basses terres tropicales périphériques apparaît comme l'image d'une frontière territoriale naturalisée à l'instar des accidents géographiques, des obstacles végétaux et des difficultés climatiques qui rendent laborieuses la pénétration et la vie dans ces contrées, l'Indien aristocratique chibcha des plateaux centraux, lui, semble être la métaphore d'une fronlière temporelle naturalisée, frontière biologisée par sa représentation en termes de «sang", entre le temps d'avant l'histoire et l'entrée dans cette dernière par l'arrivée des Espagnols.

\section{ÉCHANGGE DE REPAÉSENTATIONS ENTRE CENTAE ET PÉRPPHÉPIE :}

la nouvelle tercitorialilé colombienne

Toutefois, un changement symbolique important s'est amorcé avec la nouvelle définition de l'État comme «multi-culturel et pluri-ethnique ". Si, au niveau économique, la Colombie s'engage dans l'intégration rapide des ressources de sa périphérie territoriale dans le marché national, au niveau symbolique, la lecture des nouveaux manuels d'histoire, des documents politiques et de la presse semble attester l'émergence d'une nouvelle représentation du soi national. Elle puise certains de ses traits dans un nouveau réser- 
voir de métaphores: ainsi, l'image stéréotypée du «Noir travailleur» et celle d'un Indien générique et immémorial s'intègrent progressivement dans l'imagerie nationale du «Colombien historique» dont la caractéristique nouvelle est la «multiplicitées. L'iconographie du Président, atterrissant en divers lieux de la forêt et s'adressant en langue indienne à des représentants de sociétés jusqu'à présent ignorées, n'en est qu'un des signes massivement médiatisés.

Mais si la nouvelle représentation du «Noir travailleur" trouve facilement une assise iconographique au sein de n'importe quelle population noire de l'Occident colombien, il semblait plus difficile de concilier dans une représentation et une iconographie emblématiques les deux figuresfrontières de l'Indien : celle des hauts plateaux, ancienne et présente et celle des basses terres tropicales forestières. C'est la population Kogi de la Sierra Nevada de Santa Marta qui semble réaliser cette fusion entre passé et présent, entre l'image des Chibcha seigneuriaux et celle des Indiens forestiers: sa récente projection médiatique sur la scène de la nouvelle idéologie nationale en fait foi.

En effet, ce groupe de langue chibcha, non amazonien et non andin, occupe le cceur d"un massif montagneux mais dont la partie basse et médiane est couverte d'une forêt dense; il y conserve un système politico-religieux hiérarchisé. Sa cosmologie très intégrée et préservée est en même temps sensible à certains items du discours écologique ambiant : l'intégration de ces derniers offre aux Kogi un langage métaphorique dans lequel peut s'articuler une ouverture partielle vers la société nationale, en même temps que leur long attachement ferme à l'autonomie de leur être col- lectif. En outre, ce groupe a su faire partager à ses ethnographes sa conviction d'être les héritiers de la civilisation pré-colombienne complexe des Tairona qui a érigé en pleine forêt de montagne des "cités perdues», dont une, récemment « retrouvée», fut mise en valeur par les archéologues et partiellement ouverte au tourisme. Rien d'étonnant donc à ce que ces «Indiens du troisième type» dont l'être et le territoire semblent offrir un support fusionnel à l'intégration de tous les aspects contradictoires de l'image colombienne de l'Indien, soient en voie d'emblématisation nationale. L'inclusion de plus en plus fréquente d'une visite à la "Cité Perdue" tairona de la Sierra Nevada dans les programmes officiels des hôtes politiques étrangers, ainsi que les phrases prises dans la mythologie Kogi qui ornent les murs du nouvel aéroport international de Bogotà n'en sont que les signes les plus manifestes.

Ainsi, il apparaît qu'un échange symbolique paradoxal s'est instauré récemment entre l'espace national et les divers groupes de sa périphérie, échange dont l'enjeu est une intégration mutuelle contradictoire, cristallisée autour de l'articulation de divers types de territorialité. Si l'espace national emprunte des bribes éparses et stéréotypées du symbolisme des groupes périphériques pour refonder une légitimité plus large. ces derniers commencent à puiser dans son régime d'historicité et d'auto-représentation un type d'ethnicité et son emblématisation pour tenter de concilier leur être autonome avec leur intégration dans la modernité. Cet échange asymétrique de représentations engendre dans les sociétés minoritaires une tension nouvelle entre régimes alternatifs de mémoire et de traditions. Le caractère vertical et asymé- 
trique de ce troc symbolique se trouve à l'opposé de

la logique d'emboîtement qui a régi jusqu'à pré-

sent l'interface horizontale entre Noirs et Emberà

du Choco et va probablement modifier celle-ci.

\section{NOTES}

\section{-}

Pour l'analyse du chananisme d'agression Enherà et de ses rapports avec l'idéologie guerrière traditionnelle, voir lusoncoy [1986] : 165 et passim.

\section{$\overline{1}$}

Zone centrale de notre travail de terrain entre 1975 et 1988.

II est ì remarquer que, d'après les agronomes interrogés, l'éloignement des abattis des maisons et leur recherche sur les cours sujérieurs dos affluents n'a aucune raison d'être en termes d'une meilleure qualité de la couche fertile, donc d'in rendenent agricole pius élevé. (que les NégroColombiens le savent par expérience se prouve chaque fois que lion abandonne une habitation daus la partie basse de la riviète pour la reconstruire dans le noyau aggloméré de la Villa Claret : le termin ainsi libéré est réutilisé comme abattis, avec le même rendement que ceux du haut des cours d'ealt. (communication personnelle de 1). Caycedo at de $\mathrm{M}$. Mosquera, agronomes de l'Iniversité de (Quilydo, 1977). Il s'auit donc à n'en pas douter d un parti pris culturel de multispatialité dont les motivations se trouvent bien plus au niveau des représentations f̧u'au niveau "économique».

\section{4}

Cette assertion est corroborée par une pratique indienne consistant à utiliser dans certaines circonstances córénonielles la présence des membres de la communauté noire avec lesquels il existe une relation de parenté rituelle (compadrazgo), comme «groupe-tampon" contre la menace d'une violence physique ou symbolique (sorcellerie), dans les rapports entre limberà.

I5

P'our l'anatyse du concept Emberà du kacbirua désignant de façon générale un comportement accumnlateur qui nfuse l'échange, voir Losonczy [1986] : 164 et Vasco [1985] : 92.

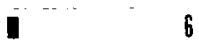

Procédés qui, d'après West [1957] et Ficalante [1971], ne survivent pas plus tels quels sur tout le continent sud-américain que dans l'Occident colombien. 